\title{
Regular fractional dissipative boundary value problems
}

\section{Dumitru Baleanu* and Ekin Uğurlu}

${ }^{\text {*} C o r r e s p o n d e n c e: ~}$ dumitru@cankaya.edu.tr Department of Mathematics, Faculty of Arts and Science, Çankaya University, Balgat, Ankara, 06530, Turkey

\begin{abstract}
In this manuscript we present a regular dissipative fractional operator associated with a fractional boundary value problem. In particular, we present two main dissipative boundary value problems and one of them contains the spectral parameter in the boundary conditions. To construct the associated dissipative operator we present a direct sum Hilbert space.
\end{abstract}

\section{Introduction}

As is well known Sturm-Liouville problems play an important role in many scientific problems. Second order Sturm-Liouville equations are of the form

$$
-\left(p(x) y^{\prime}\right)^{\prime}+q(x) y=\lambda w(x) y, \quad-\infty<a<b<\infty,
$$

where $p, q$, and $w$ are real-valued functions such that $p^{-1}, q$, and $w$ are locally integrable functions on a given interval and $w>0$ for almost all $x$. However, this classical equation can be generalized to fractional differential equation [1] with the help of left- and rightsided Riemann-Liouville integrals of order $\alpha(\operatorname{Re} \alpha>0)$ as follows [2-4]:

$$
\begin{array}{ll}
\left(I_{a^{+}}^{\alpha} y\right)(x)=\frac{1}{\Gamma(\alpha)} \int_{a}^{x}(x-s)^{\alpha-1} y(s) d s, & x>a, \\
\left(I_{b^{-}}^{\alpha} y\right)(x)=\frac{1}{\Gamma(\alpha)} \int_{x}^{b}(s-x)^{\alpha-1} y(s) d s, & x<b,
\end{array}
$$

where $\Gamma$ denotes the gamma function. Then the left-sided and right-sided RiemannLiouville derivatives of order $\alpha$ are defined as

$$
\begin{aligned}
& \left(D_{a^{+}}^{\alpha} y\right)(x)=D^{m}\left(I_{a^{+}}^{m-\alpha} y\right)(x), \quad x>a, \\
& \left(D_{b^{-}}^{\alpha} y\right)(x)=(-D)^{m}\left(I_{b^{-}}^{m-\alpha} y\right)(x), \quad x<b,
\end{aligned}
$$

where $\operatorname{Re} \alpha \in(m-1, m)$, and

$$
\begin{aligned}
& \left({ }^{c} D_{a^{+}}^{\alpha} y\right)(x)=\left(I_{a^{+}}^{m-\alpha} D^{m} y\right)(x), \quad x>a, \\
& \left({ }^{c} D_{b^{-}}^{\alpha} y\right)(x)=\left(I_{b^{-}}^{m-\alpha}(-D)^{m} y\right)(x), \quad x<b .
\end{aligned}
$$

Here, secondary ones are called the left- and right-sided Caputo derivatives of order $\alpha$.

(c) 2016 Baleanu and Uğurlu. This article is distributed under the terms of the Creative Commons Attribution 4.0 International License (http://creativecommons.org/licenses/by/4.0/), which permits unrestricted use, distribution, and reproduction in any medium, provided you give appropriate credit to the original author(s) and the source, provide a link to the Creative Commons license, and indicate if changes were made. 
Fractional calculus is one of the most useful tools to investigate the hidden properties of dynamical systems. During the last few decades a lot of developments were done in both theoretical and the applied view points [4-14], namely, some important results from classical analysis were generalized to the fractional case and fractional calculus was successfully applied to real problems which appear in science and engineering. One of the most challenging hot topics in fractional calculus is to find real world application in physics. We recall that an one-dimensional dissipative Schrödinger-type operators together with their dilations and eigenfunction expansions was discussed in [15] and it was motivated by the problems appearing in semiconductor physics (also see [16, 17]).

It is well known that [18] a linear operator $A$ acting on a Hilbert space $H$ is said to be dissipative if the imaginary part of the corresponding inner product is nonnegative, that is,

$$
\operatorname{Im}(A f, f) \geq 0, \quad f \in D(A)
$$

where $D(A)$ is the domain of the operator $A$. To investigate the spectral properties of the boundary value problems it is useful to understand the nature of the associated operator. To be more precise, we should note that if an operator associated with the boundary value problem is Hermitian (selfadjoint) then all eigenvalues of the problem are real. On the other side, an important class of nonselfadjoint operators consists of dissipative operators. For ordinary differential operator generated by an ordinary differential equation and associated boundary conditions (singular) dissipative operators have been studied in many works [16-29]. However, in fractional calculus it seems that there is no work in this field. In this paper our main aim is to construct a regular dissipative fractional operator associated with a fractional boundary value problem.

Finally, in this paper we denote by $L_{w_{\alpha}}^{2}[a, b]$ the Hilbert space consisting of all functions $y$ such that

$$
\int_{a}^{b}|y|^{2} w_{c} d x<\infty
$$

with the inner product

$$
(y, z)_{L_{w_{c}}^{2}}=\int_{a}^{b} y \bar{z} w_{c} d x
$$

\section{Boundary value problem}

As in [1], in this paper we consider the following fractional Sturm-Liouville differential expression:

$$
\mathcal{L}_{\alpha, x}:={ }^{C} D_{b^{-}}^{\alpha}\left(p(x) D_{a^{+}}^{\alpha}\right)+q(x), \quad 0<\alpha \leq 1,
$$

on the interval $[a, b]$. Here we assume that $p$ and $q$ are real-valued functions having finite values at each point on $[a, b]$ and $p(x) \neq 0$. Now we shall handle the following boundary value problem:

$$
\begin{aligned}
& \mathcal{L}_{\alpha, x} y=\lambda w_{\alpha}(x), \\
& \cos \beta I_{a^{+}}^{1-\alpha} y(a)+\sin \beta p(a) D_{a^{+}}^{\alpha} y(a)=0, \\
& I_{a^{+}}^{1-\alpha} y(b)-h p(b) D_{a^{+}}^{\alpha} y(b)=0,
\end{aligned}
$$


where $w_{\alpha}(x)$ is the real-valued function such that $w_{\alpha}(x)>0$ on $[a, b], \beta$ is a real number and $h$ is a complex number such that $h=h_{1}+i h_{2}$ with $h_{2}>0$.

Let $\mathbb{L}$ be an operator with domain $D(\mathbb{L})$, which consists of those functions $y$ such that ${ }^{c} D_{b^{-}}^{\alpha}\left(p(x) D_{a^{+}}^{\alpha} y\right)$ is meaningful satisfying (2.2), (2.3) and

$$
\frac{1}{w_{\alpha}(x)}\left(\mathcal{L}_{\alpha, x} y\right) \in L_{w_{\alpha}}^{2}[a, b]
$$

with the rule

$$
\mathbb{L} y=\frac{1}{w_{\alpha}(x)}\left(\mathcal{L}_{\alpha, x} y\right), \quad y \in D(\mathbb{L}) .
$$

Then

$$
\mathbb{L} y=\lambda y, \quad y \in D(\mathbb{L})
$$

coincides with the problem (2.1)-(2.3). Then we have the following.

Theorem 2.1 The operator $\mathbb{L}$ is dissipative in $L_{w_{\alpha}}^{2}[a, b]$.

Proof For $y \in D(\mathbb{L})$ we have

$$
(\lambda-\bar{\lambda}) w_{\alpha}(x) y \bar{y}=\mathcal{L}_{\alpha, x} y \bar{y}-\overline{\mathcal{L}_{\alpha, x} y} y
$$

and using (2.2) and (2.3) we obtain

$$
\begin{aligned}
(\mathbb{L} y, y)_{L_{w_{C}}^{2}}-(y, \mathbb{L} y)_{L_{w_{C}}^{2}}= & I_{a^{+}}^{1-\alpha} y(b) \overline{p(b) D_{a^{+}}^{\alpha} y(b)}-p(b) D_{a^{+}}^{\alpha} y(b) \overline{I_{a^{+}}^{1-\alpha} y(b)} \\
& -I_{a^{+}}^{1-\alpha} y(a) \overline{p(a) D_{a^{+}}^{\alpha} y(a)}+p(a) D_{a^{+}}^{\alpha} y(a) \overline{I_{a^{+}}^{1-\alpha} y(a)} \\
= & 2 i \operatorname{Im} h\left|p(b) D_{a^{+}}^{\alpha} y(b)\right|^{2},
\end{aligned}
$$

which implies that $\mathbb{L}$ is dissipative in $L_{w_{\alpha}}^{2}[a, b]$.

Then we arrive at the following corollary ([18], p.176).

Corollary 2.1 Let $\lambda$ be an eigenvalue of the operator $\mathbb{L}$. Then $\operatorname{Im} \lambda \geq 0$.

For the special case of $\alpha$ we have additional results [19, 20, 25-29].

Corollary 2.2 For $\alpha=1$ the boundary value problem (2.1)-(2.3) reduces to

$$
\begin{aligned}
& -\left(p(x) y^{\prime}\right)^{\prime}+q(x) y=\lambda w(x) y, \\
& \cos \beta y(a)+\sin \beta p(a) y^{\prime}(a)=0, \\
& y(b)-h p(b) y^{\prime}(b)=0 .
\end{aligned}
$$

Let $\lambda=\lambda_{0}$ be an eigenvalue of the problem (2.4)-(2.6). Then $\operatorname{Im} \lambda_{0}>0$. Moreover, the multiplicity of $\lambda_{0}$ is finite. The set of all eigenvalues are countable. All root vectors (eigen-and associated vectors) of the problem (2.4)-(2.6) span the Hilbert space $L_{w}^{2}[a, b]$. 


\section{Eigenparameter dependent boundary value problem}

The second problem is as follows:

$$
\begin{aligned}
& \mathcal{L}_{\alpha, x} y=\lambda w_{\alpha}(x) y, \\
& \gamma_{1} I_{a^{+}}^{1-\alpha} y(a)-\gamma_{2} p(a) D_{a^{+}}^{\alpha} y(a)=\lambda\left(\gamma_{1}^{\prime} I_{a^{+}}^{1-\alpha} y(a)-\gamma_{2}^{\prime} p(a) D_{a^{+}}^{\alpha} y(a)\right), \\
& I_{a^{+}}^{1-\alpha} y(b)-h p(b) D_{a^{+}}^{\alpha} y(b)=0,
\end{aligned}
$$

where $w_{\alpha}(x)$ is the real-valued function such that $w_{\alpha}(x)>0$ on $[a, b], \gamma_{1}, \gamma_{2}, \gamma_{1}^{\prime}, \gamma_{2}^{\prime}$ are real numbers such that $\gamma:=\gamma_{1}^{\prime} \gamma_{2}-\gamma_{1} \gamma_{2}^{\prime}>0$ and $h$ is a complex number such that $h=h_{1}+i h_{2}$ with $h_{2}>0$.

Let $H=L_{w}^{2}[a, b] \oplus \mathbb{C}$ be the Hilbert space with the inner product

$$
\langle Y, Z\rangle_{H}=(y, z)_{L_{w_{C}}^{2}}+\frac{1}{\gamma} y_{1} \bar{z}_{1}
$$

where

$$
Y=\left(\begin{array}{c}
y(x) \\
y_{1}
\end{array}\right), \quad Z=\left(\begin{array}{c}
z(x) \\
z_{1}
\end{array}\right) \in H,
$$

where $y_{1}=\gamma_{1}^{\prime} I_{a^{+}}^{1-\alpha} y(a)-\gamma_{2}^{\prime} p(a) D_{a^{+}}^{\alpha} y(a)$.

We construct the set $D\left(\mathbb{L}_{\lambda}\right)$ consisting of all functions $Y=\left(\begin{array}{c}y(x) \\ y_{1}\end{array}\right)$ such that $y \in L_{w}^{2}[a, b]$ satisfies the condition (3.3) with the rule

$$
\mathbb{L}_{\lambda} Y=\left(\begin{array}{c}
\frac{1}{w_{\alpha}(x)}\left(\mathcal{L}_{\alpha, x} y\right) \\
\gamma_{1} I_{a^{+}}^{1-\alpha} y(a)-\gamma_{2} p(a) D_{a^{+}}^{\alpha} y(a)
\end{array}\right) .
$$

Then the problem (3.1)-(3.3) coincides with the problem

$$
\mathbb{L}_{\lambda} Y=\lambda Y \text {. }
$$

We have the following.

Theorem 3.1 The operator $\mathbb{L}_{\lambda}$ is dissipative in $H$.

Proof For $Y \in D\left(\mathbb{L}_{\lambda}\right)$ we obtain

$$
\left\langle\mathbb{L}_{\lambda} Y, Y\right\rangle_{H}-\left\langle Y, \mathbb{L}_{\lambda} Y\right\rangle_{H}=2 i \operatorname{Im} h\left|p(b) D_{a^{+}}^{\alpha} y(b)\right|^{2} .
$$

Since $Y \in D\left(\mathbb{L}_{\lambda}\right)$ we see that $\mathbb{L}_{\lambda}$ is dissipative in $H$.

Corollary 3.1 Let $\lambda$ be an eigenvalue of the operator $\mathbb{L}_{\lambda}$. Then $\operatorname{Im} \lambda \geq 0$. For the special case of $\alpha$ we have additional results [21-24].

Corollary 3.2 For $\alpha=1$ the boundary value problem (3.1)-(3.3) reduces to

$$
\begin{aligned}
& -\left(p(x) y^{\prime}\right)^{\prime}+q(x) y=\lambda w(x) y, \\
& \gamma_{1} y(a)-\gamma_{2} p(a) y^{\prime}(a)=\lambda\left(\gamma_{1}^{\prime} y(a)-\gamma_{2}^{\prime} p(a) y^{\prime}(a)\right), \\
& y(b)-h p(b) y^{\prime}(b)=0 .
\end{aligned}
$$


Let $\lambda=\lambda_{0}$ be an eigenvalue of the problem (3.4)-(3.6). Then $\operatorname{Im} \lambda_{0}>0$. Moreover, the multiplicity of $\lambda_{0}$ is finite. The set of all eigenvalues are countable. All root vectors (eigen-and associated vectors) of the problem (2.4)-(2.6) span the Hilbert space $L_{w}^{2}[a, b] \oplus \mathbb{C}$.

\section{Conclusion}

It is well known that the dissipative operators arise in several real world applications and even naturally in mathematics. In this manuscript we considered new operators, namely they are both dissipative and of fractional calculus type. The generalization proposed in this manuscript will extend considerably the possibility to extract new features from the dynamics of complex systems involving non-local effects. Bearing this in mind we discussed first of all the boundary value problem (2.1)-(2.3) and we showed that the corresponding fractional operator $\mathbb{L}$ is dissipative in $L_{w}^{2}[a, b]$. After that we investigated the boundary value problem (3.1)-(3.3) and we proved that the corresponding operator $\mathbb{L}_{\lambda}$ is dissipative in $L_{w}^{2}[a, b] \oplus \mathbb{C}$.

Competing interests

The authors declare that they have no competing interests.

Authors' contributions

The authors equally contributed to the paper. All authors read and approved the final manuscript.

Received: 1 February 2016 Accepted: 29 May 2016 Published online: 01 July 2016

References

1. Klimek, M, Agrawal, OP: Fractional Sturm-Liouville problem. Comput. Math. Appl. 66, 795-812 (2013)

2. Samko, SG, Kilbas, AA, Marichev, Ol: Fractional Integrals and Derivatives Theory and Applications. Gordon and Breach, New York (1993)

3. Podlubny, I: Fractional Differential Equations. Academic, New York (1999)

4. Baleanu, D, Diethelm, K, Scalas, E, Trujillo, JJ: Fractional Calculus Models and Numerical Methods. Series on Complexity, Nonlinearity and Chaos. World Scientific, Singapore (2012)

5. Kilbas, A, Srivastava, HM, Trujillo, JJ: Theory and Application of Fractional Differential Equations, vol. 204, pp. 45-50. Elsevier, Amsterdam (2006)

6. Caputo, M, Fabrizio, M: Damage and fatigue described by a fractional derivative model. J. Comput. Phys. 293, 401-408 (2014)

7. Mainardi, F: Fractional Calculus and Waves in Linear Viscoelasticity, an Introduction to Mathematical Models, pp. 2-192. World Scientific, Singapore (2010)

8. Baleanu, D, Octavian, GM, Agarwal, RP: An existence result for a super linear fractional differential equation. Appl. Math. Lett. 23(9), 1129-1132 (2010)

9. Shaed, MEL: A fractional calculus model of semilunar heart valve vibrations. International Design Engineering Technical 5, 19th Biennial Conference on Mechanical Vibration and Noise, Parts A, B, and C, Chicago, Illinois, USA, September 2-6, 711-714 (2003)

10. Baleanu, D, Muslih, SI, Rabei, E: On fractional Euler-Lagrange and Hamilton equations and the fractional generalization of total time derivative. Nonlinear Dyn. 53(1-2), 67-74 (2008)

11. Baleanu, D, Muslih, SI, Tas, K: Fractional Hamiltonian analysis of higher order derivatives systems. J. Math. Phys. 47(10), Article ID 103503 (2006)

12. Baleanu, D, Avkar, T: Lagrangians with linear velocities within Riemann-Liouville fractional derivatives. Nuovo Cimento B 119(1), 73-79 (2005)

13. Zavada, P: Relativistic wave equations with fractional derivatives and pseudo differential operators. J. Appl. Math. 2(4), 164-196 (2002)

14. Fabrizio, M: Fractional rheological models for thermomechanical systems dissipation and free energies. Fract. Calc. Appl. Anal. 17(1), 206-222 (2014)

15. Kaiser, HC, Neidhardt, H, Rehberg, J: On 1-dimensional dissipative Schrödinger-type operators, their dilations and eigenfunction expansions. Math. Nachr. 252, 51-69 (2003)

16. Pavlov, BS: Dilation theory and spectral analysis of nonselfadjoint differential operators. In: Math. Programming and Related Questions (Proc. Seventh Winter School, Drogobych, 1974): Theory of Operators in Linear Spaces, pp. 3-69. Tsentral. Ekonom.-Mat. Inst. Akad. Nauk SSSR, Moscow (1976); English transl in Amer. Math. Soc. Transl. (2), vol. 115 (1980)

17. Pavlov, BS: Spectral analysis of a dissipative singular Schrödinger operator in terms of a functional model. Itogi Nauki Tekh. Ser. Sovrem. Probl. Math. Fundam. Napravleniya 65, 95-163 (1991) English transl. in Partial Differential Equations, 8, Encyc. Math. Sci., vol. 65, 1996, pp. 87-163

18. Gohberg, IC, Krein, MG: Introduction to the Theory of Linear Nonselfadjoint Operators. Am. Math. Soc., Providence (1969) 
19. Allahverdiev, BP: On dilation theory and spectral analysis of dissipative Schrödinger operators in Weyl's limit-circle case. Math. USSR, Izv. 36, 247-262 (1991)

20. Allahverdiev, BP, Canoglu, A: Spectral analysis of dissipative Schrödinger operators. Proc. R. Soc. Edinb. 127A, 1113-1121 (1997)

21. Allahverdiev, BP: A nonselfadjoint singular Sturm-Liouville problem with a spectral parameter in the boundary condition. Math. Nachr. 278(7-8), 743-755 (2005)

22. Allahverdiev, BP: A dissipative singular Sturm-Liouville problem with a spectral parameter in the boundary condition. J. Math. Anal. Appl. 316, 510-524 (2006)

23. Allahverdiev, BP, Bairamov, E, Ugurlu, E: Eigenparameter dependent Sturm-Liouville problems in boundary conditions with transmission conditions. J. Math. Anal. Appl. 401, 388-396 (2013)

24. Uğurlu, E, Bairamov, E: Spectral analysis of eigenparameter dependent boundary value transmission problems. J. Math. Anal. Appl. 413, 482-494 (2014)

25. Bairamov, E, Ugurlu, E: Krein's theorems for a dissipative boundary value transmission problem. Complex Anal. Oper. Theory 7, 831-842 (2013)

26. Bairamov, E, Ugurlu, E: On the characteristic values of the real component of a dissipative boundary value transmission problem. Appl. Math. Comput. 218, 9657-9663 (2012)

27. Uğurlu, E, Bairamov, E: Krein's theorem for the dissipative operators with finite impulsive effects. Numer. Funct. Anal. Optim. 36(2), 256-270 (2015)

28. Guseinov, GS, Tuncay, H: The determinants of perturbation connected with a dissipative Sturm-Liouville operators. J. Math. Anal. Appl. 194, 39-49 (1995)

29. Bairamov, E, Krall, AM: Dissipative operators generated by the Sturm-Liouville expression in the Weyl limit circle case. J. Math. Anal. Appl. 254, 178-190 (2001)

\section{Submit your manuscript to a SpringerOpen ${ }^{\circ}$ journal and benefit from:}

- Convenient online submission

Rigorous peer review

- Immediate publication on acceptance

- Open access: articles freely available online

- High visibility within the field

- Retaining the copyright to your article 